\title{
Metrological Properties of the Test Setup for Determination Shielding Effectiveness of the Industrial Cable Connectors
}

\author{
Ryszard Kopka, Wiesław Tarczyński, and Sławomir Pluta
}

\begin{abstract}
The paper presents results related to assessment of the repeatability and reproducibility of the measurement test setup for determination the relative value of the shielding effectiveness coefficient of the industrial connectors. The construction of the proposed test setup, the measurement method and the procedure for the analysis of measurement results were described. To determine the value of the repeatability and reproducibility coefficient, the two-way analysis of variance was used, which additionally allows for an assessment of the influence of individual sources of variance. The measurements and their analysis were conducted for several frequencies in the range up to $1 \mathrm{GHz}$. Additionally, the expanded measurement uncertainty, including the test setup and instruments uncertainty was estimated.
\end{abstract}

Keywords-shielding effectiveness, variance analysis, repeatability and reproducibility, industrial connectors.

\section{INTRODUCTION}

The development of wire and wireless communication systems, IT systems and electric devices involves the necessity of meeting higher requirements for the electronic devices in the field of electromagnetic compatibility. This is caused by the increase in the number of devices generating electromagnetic radiation in the range of frequencies from tens of hertz to dozen or so of gigahertz. To ensure proper operation of the electronic devices exposed to the adverse electromagnetic fields, protection in a form of shields is introduced, among others.

To minimize propagation of disturbances through the power systems in the electronic devices powered from the electricity network, low-pass filters are introduced aiming at preventing disturbances to spread in the range of high frequencies [1], [2].

Protection from spreading the disturbances in the range of high and very high frequencies have to include cables and connectors. The cables and connectors have a wide range of applications and occur in almost every electronic device. They are intended for sending signals, in the binary as well as - more and more often - the analog form. In all of those cases, it is very important for the transmitted signals, to be not disrupted.

One of the most popular method of protecting the signals transmitted through cables and connectors is shielding [3] [5]. It protects the cable's wires and other cable elements.

R. Kopka, W. Tarczyński and S. Płuta are with the Institute of Control and Computer Engineering, Opole University of Technology, ul. Prószkowska 76, 45-758 Opole, Poland, e-mail: \{r.kopka,w.tarczynski,s.pluta\}@po.opole.pl.
Properties of the transmitting system shield depend on the shield construction and used to its construction materials (i.e. aluminium and copper foil, braid in a form of mesh etc.) [6]-[8]. It is especially important to connect the cable and the connector shield inside the connector. It is very difficult considering the construction, especially within the connectors that are frequently connected and disconnected. In this case a compromise needs to be made between the complexity of the construction and thus the costs of construction, the shielding efficiency and its mechanical durability [9], [10].

The best method to compare the quality of the cable and connector shielding is co called shielding effectiveness, defined as a ratio of the signal induced in the cable and connector wires, to the surrounding signal [9], [11]. The literature describes a lot of systems and methods of measuring the effectiveness of connectors shielding along with the wires [4], [11]. The main disadvantage of these systems and measuring method is using a flexible wire mounted along the cable and connectors as the radiating source. Such construction of the measuring circuit results in low repeatability of the measurement conditions. The next disadvantage is that the fields influence each other also outside the connector. This can cause additional measurement errors. In such situation, the disturbing signal, especially in the range of very high frequencies, spreads from the source to the parts of the measuring device laying outside the zone of measurement.

The controlled repeatability of the measurement conditions has especially significant meaning in the case of executing the control measurements during connector prototyping and introducing the new solutions. There is a need of control the influence of the construction changes on the connector properties.

The paper presents the results of the tests the properties of the designed setup intendent to measure the effectiveness of the connectors and cable shielding, ensuring very high repeatability of the measuring conditions. Such setup allows to observe and assess the effect of the construction solution and the material changes on the effectiveness of the connector and wires shielding as so as the process quality [12], [13].

The paper comprises of 5 chapters. The chapter 2 describes the basic theoretical issues related to the assessment of the shielding effectiveness, the definitions of the main quantities describing electromagnetic tests of the connectors and the properties of the test setup. In chapter 3 the construction 
of the proposed setup and the methods of preparation and realization of the measurements are described. Chapter 4 presents the results of measurements of the connector shielding effectiveness and the assessment of the metrological properties of the measuring system. Chapter 5 provides a short summary of the obtained results and currently performed works related to preparation the new measurement system.

\section{THE MEASURING PROCEDURES AND THE METHODS OF ASSESSING THE SHIELDING EFFECTIVENESS}

The basic parameters used for the assessment of the quality of the connector shield are: the shielding effectiveness $-\alpha_{S E}$, transfer impedance $-Z_{T}$ and selected elements of dispersion matrix - S [11], [14], [15]. These parameters and the methods for them measuring were defined for the frequency range for which the dimensions of the connector are much lower than the wavelength of the test signal.

The shielding effectiveness $\alpha_{S E}$ is defined as the ration of the signal value passes through the shield $E_{T}$ to the value of this signal incident at this shield $E_{I}$. The signal can be expressed as the value of the intensity of the electric or magnetic field, or as its power density. In the case of the shield assessment based on the electric field intensity, the shielding effectiveness will be defined as:

$$
\alpha_{S E}=20 \log _{10} \frac{E_{T}}{E_{I}} \quad[\mathrm{~dB}]
$$

where $E_{I}$ is the intensity of an electric field incident on the tested element, and $E_{T}$ is the value of the field in the space between the internal conductor and the shield [16]. The

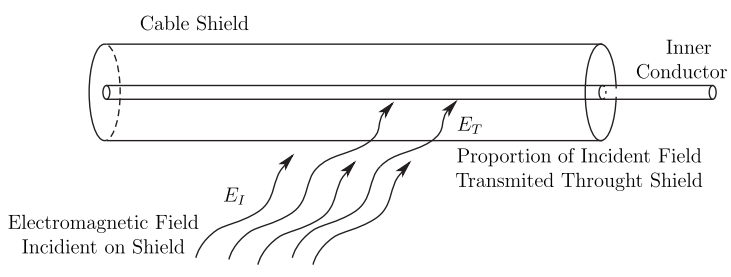

Fig. 1. The definition of the shielding effectiveness coefficient $\alpha_{S E}$.

measurement of the shields effectiveness of the cables and connectors according to the Eq. 1 is difficult to realize because it requires to measure the intensity of an electric field between the shield and the internal conductor. That is why the shielding effectiveness defined according to this definition can be mainly used to determine the properties of the flat shields.

In practice, for the assessment of the shielding effectiveness of the cable and connector, the method being the modification of the test setup and the procedure presented in the Fig. 1 is used [17]. Namely, the power of the signal induced in the internal conductor of the connector $P_{T}$ and the output power of the generator $P_{I}$ are measured, while the output power is provided to the radiating element generating the electromagnetic field in direct proximity of the shield. The shielding effectiveness defined for exactly this measuring device will be then determined as:

$$
\alpha_{S E}=10 \log _{10} \frac{P_{T}}{P_{I}} \quad[\mathrm{~dB}] .
$$

If we assume that all conditions of the wave adjustment are met, and the $P_{I}$ is equal $P_{G}$ - the output power of signal generator, and $P_{T}$ is equal $P_{V S A}$ - the power measure on the input of the vector signal analyser, then, using the decibel scale, it can be assumed that the shielding effectiveness can be calculated as:

$$
\alpha_{S E[\mathrm{~dB}]} \approx P_{T[\mathrm{dBm}]}-P_{I[\mathrm{dBm}]}=P_{V S A[\mathrm{dBm}]}-P_{G[\mathrm{dBm}]} .
$$

The test setup according to this method is shown in the Fig. 2. The disadvantage of this method is very low accuracy resulting from the difficulty accurately determine the part of the power of the signal provided by the generator, passes through the tested area and what part is being lost in the cables delivering the signal, is dispersed at the connections, unfitted areas etc. In addition, a numerous measurements have shown that the reciprocal alignment of the tested connectors and the radiating element, and additionally their joint alignment against the surroundings, have a significant influence on the measurement results [10].

Lack of unambiguously specified alignment between the radiating and testing elements, and conditions of performing the measurements causes that the obtained results are incomparable between each other and thus making it impossible to assess the real properties of the shield. That is why a concept of the relative shielding effectiveness coefficient have been introduced $-\alpha_{S E}^{\prime}$, indicating reference of its value to the certain measuring setup. Whereas an advantage of this method is the possibility of performing the measurements in a simple system, the possibility of using typical instruments, and that is why this method is preferred to be used in the assessment of changes of the connectors and cables properties on the development of new construction or in the process of control of the serial production quality.

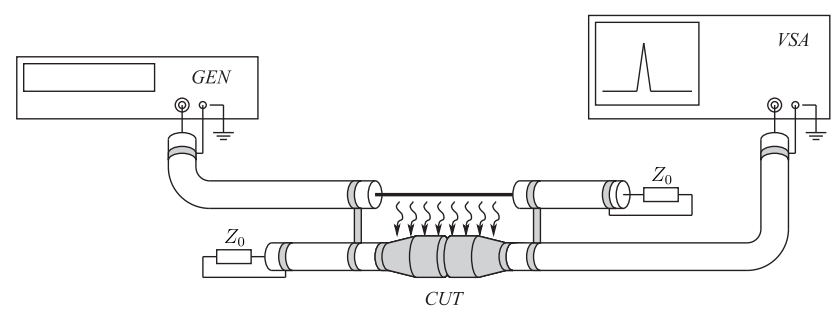

Fig. 2. The test setup for measuring the relatively value of shielding effectiveness coefficient $\alpha_{S E}^{\prime}$ by measuring the input and output power (GEN-Generator, VSA-Vector Signal Analyser).

The assessment of the shield properties by the measurement of the elements of the dispersion matrix $\mathbf{S}$ is being performed using the voltage wave as the ratio of the wave incident on the connectors of the circuit to the reflected [3]. The measurement is performed in the setup as presented in the Fig. 2. The values of each element of the dispersion matrix $\mathbf{S}$ are determined using equation:

$$
\left[\begin{array}{l}
V_{1}^{-} \\
V_{2}^{-}
\end{array}\right]=\left[\begin{array}{ll}
S_{11} & S_{12} \\
S_{21} & S_{22}
\end{array}\right]\left[\begin{array}{l}
V_{1}^{+} \\
V_{2}^{+}
\end{array}\right]
$$


where: $\mathbf{V}^{+}$is a vector of the voltage waves incident on connectors, and $\mathbf{V}^{-}$is a vector of the amplitudes of waves getting out of the connectors. In this case, the value of the connector shielding effectiveness is determined by the value of the coefficient $S_{21}$ or $S_{12}$ (Fig. 3). In the properly

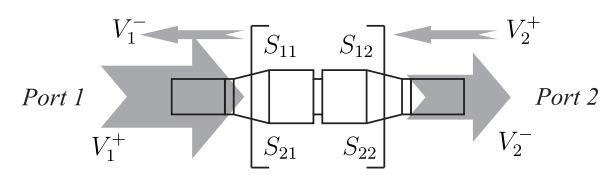

Fig. 3. The dispersion matrix elements used to definition the shielding effectiveness.

set measuring circuit, the values should fulfil the formula: $S_{11}=S_{22}=0$ and $S_{21}=S_{12}$. The conditions $S_{11}=0$ and $S_{22}=0$ mean matching the waves on the input and output terminal, and the condition $S_{21}=S_{12}$ means that the circuit is non-directional. With the ideal shielding, the value of the coefficients $S_{12}$ and $S_{21}$ should tend to infinity [3].

The properties of the shield are also assessed through the surface transfer impedance $Z_{T}$ that is defined as a ratio of the voltage drop $U$ on the shield length $l$, to the current value $I$ that was caused by the voltage drop and is expressed as the unit of length [11]. The advantage of this method is that measurement is independent of the test setup. This means that any test method may be used to determine the transfer impedance and the test results should be comparable with the data obtained on the same connector in a different test setup. It can be used as some kind of a reference setup in relation to other measuring equipment and methods. The disadvantage of the assessment of the shield properties using this method is that it can be used for the measurement in the frequency rage not higher than $200 \mathrm{MHz}$, because the results obtained for higher frequencies are influenced by other phenomenons, e.g. the skin effect, phenomenon of the long line, and other. The total length of the tested element should not exceed tenth part of the signal wavelength used to conduct the measurement [4]. The measurement of the shielding effectiveness according to this definition almost never can be used for testing the connectors and cables in which the shields do not have galvanic connection. Due to the way of conducting the measurement, this method will not be used for assessing the quality of the constructed test setup.

In our test the relative value of the coefficient of the shielding effectiveness was used to evaluate and compare the quality of all connectors. Its value was determined on the basis of the measurement of the elements of the dispersion matrix S.

\section{Construction of the test Setup}

Making the measuring circuit we mostly followed the recommendations contained in the standard [17]. The construction scheme is shown in the Fig. 4, while the Fig. 4(a) shows the side view, and the Fig. 4(b) the cross-section A-A. The test setup is intended to measure the ICT connector in the frequency range from a few $\mathrm{kHz}$ to single $\mathrm{GHz}$. The main advantage of the constructed test setup is ensuring high stability and repeatability of the fixing of the tested connector with the cables during measurements. It is especially significant during tests of the cable and connector quality and during the develop of new constructions and in a case of conducting control measurements during the serial production. The measuring circuit comprises of two supports $S$ that are fastened with screws to the main base $M B$. The gap between the supports $S$ is set by the standard [17] and it is equal $10 \mathrm{~cm}$, while for the connectors of bigger sizes the necessity of increasing the gap cannot be excluded. The task of the main base $M B$ is to ensure the stiffness of the measuring circuit, ensuring fixed gaps between the elements of the measuring circuit and making the conditions of the measurement independent from the influence of the surroundings, especially the ground. To the supports $S$ additionally two vertical screens $S C$ are fastened. The intend of these screens is separating the area of the generator side and the area of the measuring device side from the area of actual measurement of the connector for the signals of high frequency. Separating those areas with the screens $S C$, decreases the penetrating of the unwanted signals of high frequency through the free space, and, at the same time, ensures unambiguous separation of the testing measurement zone of the connector CUT and its wiring $W$.

The tested connector $C U T$ along with the wiring $W$ is fixed on the supports $S$. In the upper part of every support, a recess is made in which the connector cable is to be put. In the upper part of every support, crossbars $C B$ are inserted that are fastened to the supports $S$ with screws. On the cables $W$, on both sides of the connector CUT in a spot of their fixing in the recess, the external insulation is removed until the level of the shielding wire $S W$ on the length of about $1 \mathrm{~cm}$. In the crossbar, over the recess, there is a clamping screw $C S$ for clamping the support $S$ for the time of the measurement of the shielding wire of the cable $S W$. Under the recess $S$ of every support, there is a hole in which the coaxial connector is embedded. On the side of the generator, through the coaxial cable, signal is provided to the radiating element, and to the coaxial connector, a short coaxial cable ending with the adjusting circuit $A$ is connected on the other side of the measuring circuit. The adjusting circuits $A$ and $A C$ are constructed in a form of a metal recess closed with a cover. The signal is provided to the inside through the coaxial connector. Inside, the adjusting resistors are installed. In a case of testing the multi-core connectors, all of the wires in the adjusting circuits are connected together and limited by one resistor. The wave impedance of the multi-core cable with wires connected parallelly is experimentally determined with the impulse methods by measuring the amplitude of impulses propagating in it [18], [19].

The tested connector $C U T$ along with the cables $W$ is placed between the supports $S$, on about $4 \mathrm{~cm}$ height over the main base $M B$. The cable $W$ end is ended with the adjusting circuit $A$ and the other end - with the adjusting circuit $A C$ to ensure adjustment of the wave impedance of the connector $C U T$ cable 


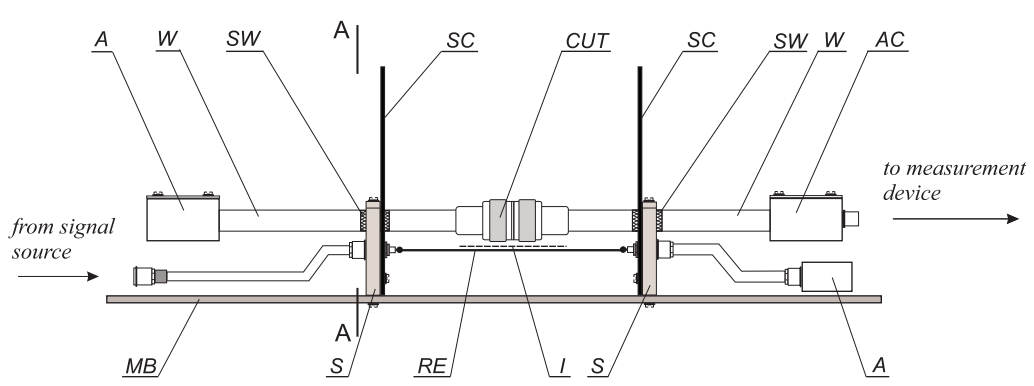

(a) side view

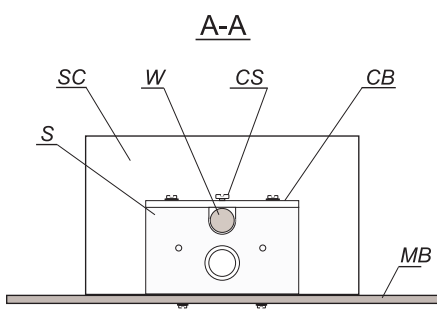

(b) the A-A cross section

Fig. 4. The test setup scheme.

to the impedance of the coaxial cable providing the signal to the measuring device. Between the middle wires of the coaxial connectors, the radiating element $R E$ made of copper tape is set. Its shape is constructed in such a way that it adheres to the connector cables and to the connector itself. In a case when the connector is made of metal, in order to ensure galvanic separation, a thin layer of insulation $I$ is inserted between the connector and the radiating element. According to the reports [16], [17], the relative value of the shielding effectiveness when proper conditions are met concerning the adjustment impedance, can be determined as:

$$
\begin{aligned}
\alpha_{S E[d B]}^{\prime} & =20 \log _{10} \frac{U_{R S A[m V]}}{U_{G[m V]}}= \\
& =20 \log _{10} \frac{\sqrt{P_{R S A[m W]} Z_{0}}}{\sqrt{P_{G[m W]} Z_{0}}}= \\
& =10 \log _{10} \frac{P_{R S A[m W]}}{P_{G[m W]}}= \\
& =P_{R S A[d B m]}-P_{G[\mathrm{dBm}]}
\end{aligned}
$$

in the frequency range up to $1 \mathrm{GHz}$.

\section{ANALYSIS OF REPEATABILITY AND REPRODUCIBILITY}

The metrological characteristics of the developed test setup in regards to the producers needs, concern ensuring the repeatability and reproducibility related to the determination of the values of the relative shielding effectiveness coefficient of the produced connectors. In this case, the repeatability is defined as the variance resulting from multiple measurements of the same element in the same test setup by the same appraiser. The reproducibility is the variance of the average value resulting from conducting the tests by different appraisers [20].

The advantage of such analysis is that it is not required to know the values of the uncertainty contributed by each measuring devices. The algorithm of the measuring procedure itself ensures that these uncertainties, although unknown, will be included in the final result which is the assessment of the percentage value of the common factor, calculated as the result of the combined influence of all of the random and systematic uncertainties [21].

The literature reports several methods of conducting the procedure of the assessment of the repeatability and reproducibility. These methods differ between each other in regards to the complexity of calculations, sensitivity, and the amount of conclusions that can be drawn from them. In this paper, the assessment bases on the variance analysis (ANOVA), more precisely on the two-way analysis of variance with constant model with repetitions. This method is the most accurate, and it additionally allows to determine the interaction between the elements being the sources of the variance. Similarly to the other methods, it bases on the statistical inference assessing the influence of different conditions on the experiment result. The sources of variance and the procedures of calculating each elements to assess the developed test setup are shown in the Table I.

The two-way analysis of variance including the interaction between the appraiser and the measured element allows testing of three statistical hypothesis:

1) $H_{0}$ : all of the tested connectors are the same against the hypothesis $H_{1}$ : all of the tested connectors are not the same;

2) $H_{0}$ : every appraisers are as precise as all the others against the hypothesis $H_{1}$ : not every appraisers are as precise as all the other;

3) $H_{0}$ : interaction between the appraiser and the tested connector can be omitted against the hypothesis $H_{1}$ : interaction between the appraiser and the tested connector cannot be omitted.

High value of the $F$ statistics usually allows to reject the zero hypothesis while its low value indicates lack of basis for the rejection. The precise decision is made on the basis of the probability $p$ of the statistics $F$ (the sixth column of the Table III) for the assumed significance level $\alpha$ and the number of the degrees of freedom $d f$.

Based on such determined elements of the analysis of variance, the value of the required repeatability and reproducibility of the test setup can be estimated. The repeatability is defined as:

$$
\sigma_{\text {repeatability }}=\kappa_{\alpha} \sqrt{M S E},
$$

while the system reproducibility as:

$$
\sigma_{\text {reproducability }}=\kappa_{\alpha} \sqrt{\frac{M S A-M S A B}{b n}} .
$$


TABLE I

THE ANOVA TABLE AND HYPOTHESIS TEST.

\begin{tabular}{lcccc}
\hline source of variation & sum of squares & degree of freedom & mean square & $F$-ratio \\
\hline appraiser & $S S A$ & $a-1$ & $M S A=S S A /(a-1)$ & $F=M S A / M S E$ \\
part & $S S B$ & $b-1$ & $M S B=S S A /(b-1)$ & $F=M S B / M S E$ \\
interaction & $S S A B$ & $(a-1)(b-1)$ & $M S A B=S S A B /((a-1)(b-1))$ & $F=M S A B / M S E$ \\
equipment & $S S E$ & $a b(n-1)$ & $M S E=S S E /(a b(n-1))$ & $\times$ \\
total & $T S S$ & $N-1$ & $\times$ & $\times$ \\
\hline
\end{tabular}

where: $a$ - is the number of appraisers, $b$ - is the number of parts, $n-$ is the number of trials, and $S S A=\sum_{i=1}^{a}\left(x_{i} . .\right)^{2} / b n-(x \ldots)^{2} / N$, $S S B=\sum_{j=1}^{b}\left(x_{\cdot j \cdot}\right)^{2} / a n-(x \ldots)^{2} / N, S S A B=\sum_{i=1}^{a} \sum_{j=1}^{b}\left(x_{i j} \cdot\right)^{2} / n-(x \ldots)^{2} / N-S S A-S S B$,

$S S E=T S S-S S A-S S B-S S A B, T S S=\sum_{i=1}^{a} \sum_{j=1}^{b} \sum_{k=1}^{n}\left(x_{i j k}\right)^{2}-(x \ldots)^{2} / N, x \ldots=\sum_{i=1}^{a} \sum_{j=1}^{b} \sum_{k=1}^{n} x_{i j k}, N=a+b+n$.

The interaction between the appraiser and the tested connector as:

$$
\sigma_{\text {interaction }}=\kappa_{\alpha} \sqrt{\frac{M S A B-M S E}{n}},
$$

and the variability of the measurement for a single connector as:

$$
\sigma_{\text {part }}=\kappa_{\alpha} \sqrt{\frac{M S B-M S A B}{a n}} .
$$

For all of the mentioned calculations, the value of the $\kappa$ parameter is calculated, assuming certain significance level $\alpha$, and assuming normal distribution, then:

$$
\kappa_{\alpha}=2 u_{1-\alpha / 2},
$$

and taking $\alpha=1 \%$, we receive $\kappa_{0.01}=5.15$.

The test setup repeatability and reproducibility $\left(\sigma_{R \& R}\right)$ is the combined estimate of the measurement system repeatability and reproducibility variance components. This is determined as:

$$
\sigma_{R \& R}=\sqrt{\sigma_{\text {repeatability }}^{2}+\sigma_{\text {reproducability }}^{2}+\sigma_{\text {interaction }}^{2}} .
$$

Within the manufacturing environment, $\sigma_{R \& R}$ error is added into the product distribution as a pure error term. This has the effect of widening the true product distribution by this amount. Representing the true product distribution as $\Delta y=\sigma_{\text {part }}$, the resulting total variation - $\sigma_{\text {totalvariation }}$ of the manufacturing distribution is determined as:

$$
\sigma_{\text {totalvariation }}=\sqrt{\sigma_{R \& R}^{2}+\sigma_{\text {part }}^{2}} .
$$

With a knowledge of the components of the total variation, some useful performance metrics for the measurement system can be generated. The percentage of total variation $\sigma_{R \& R[\%]}$ is used the most commonly. These is calculated as:

$$
\sigma_{R \& R[\%]}=\frac{\sigma_{R \& R}}{\sigma_{\text {totalvariation }}} \times 100 \% .
$$

This metric gives an indication of how capable the test setup is for measuring the critical parameters to quality characteristic. Acceptable regions of measurement system based on $\sigma_{R \& R[\%]}$ error is as indicated in Table II.
TABLE II

ACCEPTABLE RANGES OF MEASUREMENT SYSTEM $\sigma_{R \& R[\%]}$ ERROR.

\begin{tabular}{ccc}
\hline & $\sigma_{R \& R[\%]}$ range & Action required \\
\hline$\sigma_{R \& R[\%]}<10 \%$ & measurement system acceptable \\
$10 \% \leq \quad \sigma_{R \& R[\%]}<30 \%$ & action required \\
& & to understand the variance \\
$30 \% \leq \quad \sigma_{R \& R[\%]}$ & setup unacceptable for use \\
& & and requiring improvement \\
\hline
\end{tabular}

According to the Eq. 5 the value of the relative shielding effectiveness coefficient $\alpha_{S E}^{\prime}$ can be determined as:

$$
\alpha_{S E}^{\prime}=P_{G[d B m]}-P_{R S A[d B m]} .
$$

Expressing the measurement result as:

$$
y=\bar{y} \pm U=\bar{y} \pm k_{\alpha} u_{c y},
$$

where $\bar{y}$ means the average value, while $U$ the expanded uncertainty obtained by multiplying the combined standard uncertainty $u_{c y}$ by a coverage factor $k_{\alpha}$. Its value, is chosen on the basis of the required confidence level. Considering probability distribution of $y$ and $u_{c y}$ is approximately normal and the effective degrees of freedom of $u_{c y}$ is of significant size, one can assume that taking $k_{\alpha}=2$ produces an interval having a level of confidence of approximately $95 \%$. Assuming according to the Eq. 14 indirect measuring manner, i.e.:

$$
y=f\left(x_{i}\right), i=1,2, \ldots n,
$$

the value of the standard measurement uncertainty $u_{c y}$ was estimated on the basis of the equation:

$$
u_{c y}=\sqrt{\sum_{i=1}^{k}\left(\left.\frac{\partial y}{\partial x_{i}}\right|_{x_{i}} u_{x_{i}}\right)^{2}},
$$

where the relative standard uncertainty $u_{x_{i}}$ of every measured value $x_{i}$ was determined as:

$$
u_{x_{i}}=\sqrt{\left(u_{x_{i}}^{A}\right)^{2}+\left(u_{x_{i}}^{B}\right)^{2}} .
$$


In this formula $u_{x_{i}}^{A}$ and $u_{x_{i}}^{B}$ means the standard uncertainty related to the random (incidental) and the regular condition, respectively. The random part was estimated on the basis of a series of measurements carried out as:

$$
u_{x_{i}}^{A}=S_{\bar{x}} .
$$

The uncertainty of the average value of the measurement $S_{\bar{x}}$ is defined as $S_{\bar{x}}=S_{x} / \sqrt{n}$, while the $S_{x}$ is calculated as:

$$
S_{x}=\sqrt{\frac{1}{n-1} \sum_{i=1}^{n}\left(\bar{x}-x_{i}\right)^{2}}
$$

The inaccuracy of measuring devices was estimated on the basis of their systematic error $\xi$ and the assumed model of the probability distribution. Assuming the rectangular distribution with the parameter $\alpha=\xi_{\max }$, the value of the systematic error $u_{x_{i}}^{B}$ can be calculated as:

$$
u_{x_{i}}^{B}=\frac{\xi_{\max }}{\sqrt{3}},
$$

where the maximum value of the error $\xi_{\max }$ was determined based on the manufacturers specification.

\section{AnAlysis the Test Results}

The test procedure involved determination of the value of the relative shielding effectiveness coefficient $\alpha_{S E}^{\prime}$ of the shield for three simple connectors of the M12 type, and the assessment of the repeatability and reproducibility $\sigma_{R \& R[\%]}$ of the testing setup. The measurements were conducted for 5 frequencies: $50 \mathrm{MHz}, 150 \mathrm{MHz}, 400 \mathrm{MHz}, 800 \mathrm{MHz}$, and $1 \mathrm{GHz}$. For every connector and for every frequency, the measurements were performed 10 times. The measurements were conducted in a sequential manner, i.e. after fixing the first connector in the measuring setup and measuring the relative shielding effectiveness coefficient for all frequencies, the first connector was removed from the setup and the second one was fixed. After performing measurements for the second connector, it was removed and the third one was fixed. Such sequence comprised one measuring procedure After ending one procedure, first connector was once again fixed on the test setup. During the whole cycle of the test, the abovementioned procedure was repeated ten times by one employee, and three employees took part in the whole test cycle. In the Fig. 5, the results of measuring the relative shielding effectiveness coefficient $\alpha_{S E}^{\prime}$ for three connectors performed by three employees, obtained for four frequencies, are presented. Analysis of the results clearly indicates high variability in the average values for individual connectors. Despite the fact that they originated from one technological process, the differences in the measured value of the relative shielding effectiveness coefficient of individual items can be clearly noticed. The measurements results with the estimated value of the extended measurement uncertainty including the random and systematic uncertainties are shown in the Table IV, in column 3 and 4 , respectively.

The assessment of the repeatability and reproducibility of the proposed measuring procedure and the constructed test setup was conducted according to the chapter 4 , based on the two-way analysis of variance (ANOVA). Examples of the results of the analysis of variance for the frequency $f=50 \mathrm{MHz}$ are shown in Table III. This data also confirm that the main source of variance are the differences in the properties of the connectors itself (high value of the $F$ statistic).

On the basis of the results of the analysis of variance according to Eq. 6 and 7, the indicators of the repeatability and reproducibility was determined for the measuring procedure and the test setup that were compared to the total variance determined by the Eq. 12. The results for all of the frequencies are contained in the Table IV, in column 5 and 6, respectively. In column 7 , the results of the indicator cumulatively assessing the repeatability and reproducibility of the method calculated in accordance with the Eq. 13.

TABLE IV

COMPARISON OF MEASUREMENT RESULTS OF THE $\alpha_{S E}^{\prime}$ AND THE REPEATABILITY AND REPRODUCIBILITY OF THE MEASURING PROCEDURE AND TEST SETUP FOR DIFFERENT FREQUENCIES $f$.

\begin{tabular}{cccccc}
\hline $\begin{array}{c}f \\
{[\mathrm{MHz}]}\end{array}$ & $\begin{array}{c}\bar{y}\left(\bar{\alpha}_{S E}^{\prime}\right) \\
{[\mathrm{dB}]}\end{array}$ & $\begin{array}{c}U_{95}\left(k_{\alpha}=2\right) \\
{[\mathrm{dB}]}\end{array}$ & $\begin{array}{c}\sigma_{R e p e} \\
{[\%]}\end{array}$ & $\begin{array}{c}\sigma_{R e p r} \\
{[\%]}\end{array}$ & $\begin{array}{c}\sigma_{R \& R} \\
{[\%]}\end{array}$ \\
\hline 50 & -41.49 & 1.40 & 7.59 & 3.95 & 9.50 \\
150 & -42.74 & 1.09 & 17.80 & - & 18.35 \\
400 & -46.28 & 1.51 & 35.41 & 5.85 & 36.46 \\
800 & -44.18 & 2.10 & 19.92 & - & 21.81 \\
1000 & -40.31 & 1.52 & 16.53 & 3.79 & 19.13 \\
\hline
\end{tabular}

\section{CONCLUSIONS}

The article presents the results of the assessment of the repeatability and reproducibility of the developed test setup intended for determination of the relative shielding efficiency coefficient of the industrial connectors. This type of research is of great importance in the production process in terms of production quality assessments and the validation of new construction and technology solutions, as well as the new materials introduction. The quality of the test setup was assessed using the two-way analysis of variance. That is one of the methods recommended in the Measurement Systems Analysis procedures. The analysis of the results indicates high dependence of the accuracy and the value of repeatability and reproducibility on the frequency. This confirms the presence of different wave phenomena for which the construction of connectors or the entire test setup leads to reflected or standing waves or their interferences. Further work is required to allow for improving the measuring properties, especially for 400 $\mathrm{MHz}$ frequency signals. Only ensuring proper conditions of repeatability and reproducibility of the measurements procedure allows reliable assess the modifications of construction and materials introduced to the tested product during the manufacturing process.

The presented works concerning to develop setup for testing typical connections. In similar way, also tests setup for the angular connectors and signal splitters are being consider. 

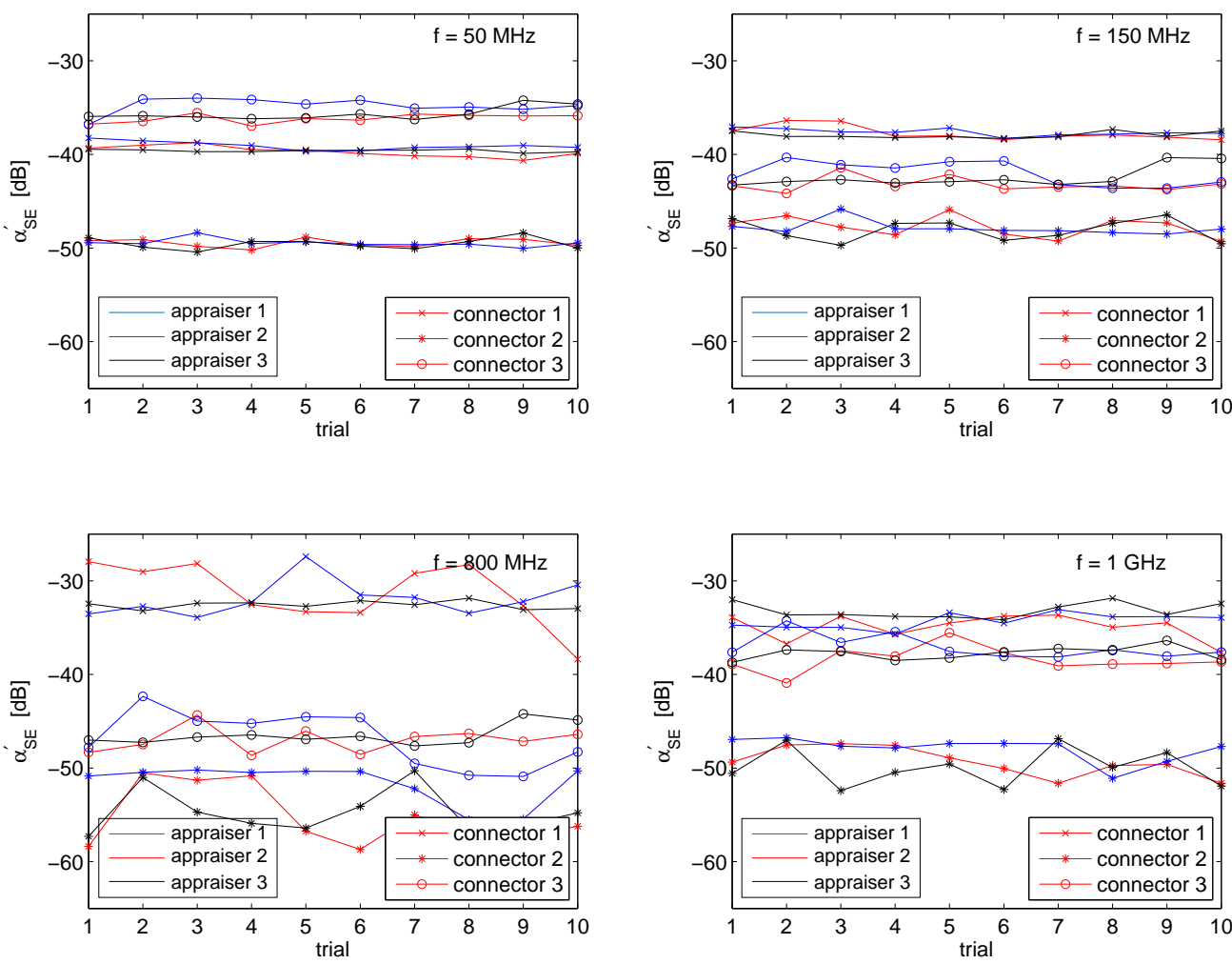

Fig. 5. Changes in the values of the relative shielding effectiveness coefficient $\alpha_{S E}^{\prime}$ of three connectors, received during the measuring procedures conducted by three appraisers for four frequencies $50 \mathrm{MHz}, 150 \mathrm{MHz}, 800 \mathrm{MHz}$ and $1 \mathrm{GHz}$, respectively.

TABLE III

THE TWO-WAY ANOVA RESULTS OF THE MEASUREMENTS OF THE $\alpha_{S E}^{\prime}$ FOR THE FREQUENCY $f=50$ MHZ.

\begin{tabular}{lcccccc}
\hline source of variation & sum of squares & degree of freedom & average square & statistic $F$ & $p$ value & $F$-critical $(p=0.05)$ \\
\hline appraiser & 7.26 & 2 & 3.63 & 12.09 & $2.54 \times 10^{-5}$ & 3.11 \\
connector & 3104.93 & 2 & 1552.46 & 5168.49 & $3.74 \times 10^{-86}$ & 3.11 \\
interaction & 4.77 & 4 & 1.19 & 3.97 & 0.0054 & $\times$ \\
equipment & 24.33 & 81 & 0.30 & $\times$ & $\times$ & $\times$ \\
total & 3141.29 & 89 & $\times$ & $\times$ & $\times$ & $\times$ \\
\hline
\end{tabular}

These elements require using different construction solutions as well as electric connections.

\section{REFERENCES}

[1] P. R. Clayton, Introduction to Electromagnetic Compatibility. New York: John Wiley \& Sons, Inc., 2006.

[2] K.-H. Gonschorek and R. Vick, Electromagnetic Compatibility for Device Design and System Integration. Berlin Heidelberg: SpringerVerlang, 2009.

[3] R. Sorrentino and G. Bianchi, Microwave and RF Engineering. Chichester: John Wiley \& Sons, Ltd., 2010.

[4] A. Tsaliovich, Cable Shielding for Electromagnetic Compatibility. New York: Chapman \& Hall, 1995.

[5] H. W. Ott, Noise Reduction Techniques in Electronic Systems. New York: John Wiley \& Sons, Inc., 1988.

[6] M. Golio and J. Golio, RF and Microwave Passive and Active Technologies. Boca Raton: Taylor \& Francis Group, 2008.

[7] L. Nowosielski, M. Wnuk, R. Przesmycki, K. Piwowarczyk, and M. Bugaj, "Coaxial cables shielding efficiency measuring methodology," in Progress In Electromagnetics Research Symposium (PIERS 2012), 27-30 March, 2012, KL, Malaysia, 2012, pp. 72-76.
[8] D. M. Pozar, Microwave Engineering. Hoboken: John Wiley \& Sons, Inc., 2012.

[9] W. J. Krzysztofik, R. Borowiec, and B. Bieda, "Some consideration on shielding effectiveness testing by means of the nested reverberation chambers," Radioengineering, vol. 20, no. 4, pp. 766-774, 2011.

[10] Q. Zhang, L.-H. Shi, Y. H. Zhou, C. Gaoand, and Y. C. Guo, "Measurement of the shielding effectiveness of connector by improved triaxial method," in Progress In Electromagnetics Research Symposium (PIERS 2011), 12-16 September, 2011, Suzhou, China, 2011, pp. 1334-1338.

[11] L. O. Hoeft and J. S. Hofstra, "Measured electromagnetic shielding performance of commonly used cables and connectors," IEEE Transactions on Electromagnetic Compatibility, vol. 30, no. 3, pp. 260-275, 1988.

[12] W. Tarczyński and R. Kopka, "A system for testing data communications connectors with cable," PL Patent Application P.409 190, 08 18, 2014

[13] _ _ "A system for investigation the ICT connectors with cable," PL Patent Application P.410 237, 11 24, 2014.

[14] T. Kleine-Ostmann, K. Münter, and T. Schrader, "A new shielding effectiveness measurement method based on a skin-effect transmission line coupler," Advances in Radio Science, no. 5, pp. 37-42, 2007.

[15] W. T. Więckowski and M. J. Janukiewicz, "Methods for evaluating the shielding effectiveness of textiles," FIBRES \& TEXTILES in Eastern Europe, vol. 14, no. 5, pp. 18-22, 2006. 
[16] J. W. Gooch and J. K. Daher, Electromagnetic Shielding and Corrosion Protection for Aerospace Vehicles. LLC: Springer Science+Business Media, 2007.

[17] "Electromechanical components for electronic equipment - Basic testing procedures and measuring methods - Part 23-3: Test 23c: Shielding effectiveness of connectors and accessories." International Organization for Standardization, Geneva, CH, Standard, 12012.

[18] W. Tarczyński, "Pulse method measurements for determination of line fault location," Archives of Electrical Engineering, vol. LV, no. 3-4, pp. 301-312, 2006.
[19] —-, "Mutual interaction of pulses in three-conductor coupled lines," Archives of Electrical Engineering, vol. LVI, no. 1, pp. 79-87, 2007.

[20] "Accuracy (trueness and precision) of measurement methods and results. Part 1: General principles and definitions." International Organization for Standardization, Geneva, CH, Standard, 11994.

[21] "Accuracy (trueness and precision) of measurement methods and results. Part 2: Basic method for the determination of repeatability and reproducibility of a standard measurement method." International Organization for Standardization, Geneva, CH, Standard, 11994. 